\title{
The Evolution of Galaxies in Clusters
}

\author{
John Dubinski \\ Department of Astronomy and Astrophysics/CITA, McLennan Labs, 60 \\ St. George St., University of Toronto, Toronto, ON, Canada M5S $3 \mathrm{H} 8$ \\ Dan Koranyi and Margaret Geller \\ Harvard-Smithsonian Centre for Astrophysics, 60 Garden St., \\ Cambridge, MA, USA 02138
}

\begin{abstract}
We report on recent numerical investigations of the dynamical evolution of galaxies in clusters. Simulations of spiral galaxies falling into forming clusters show the development of the morphology-density relationship and the formation of regular and giant elliptical galaxies. The regular elliptical merger remnants end up in a fundamental plane very similar to the observed relation. The giant ellipticals have much in common with their real counterparts but their central velocity dispersions are too high. We also quantify the amount and distribution of diffuse light in clusters.
\end{abstract}

\section{Introduction}

As pointed out by Dressler years ago, galaxy clusters can be thought of as laboratories for studying galaxy formation (Dressler 1984). There are distinct differences between galaxy populations in the field and those within clusters. First, elliptical galaxies are much more abundant within the centers of clusters and more generally in regions of high galactic surface density. This is commonly known as the morphology-density effect (Dressler 1980). The conditions within clusters during their collapse and formation seem to be conducive to the formation of large numbers of ellipticals which for the most part are not so common in the field population. Second, galaxy clusters and groups almost without exception harbour a giant elliptical galaxy near their center. These giant ellipticals have been studied in detail and they are sometimes found to deviate from $R^{1 / 4}$ light profiles at large radii in the $\mathrm{cD}$ galaxies. The giant ellipticals are also telling us something about galaxy and cluster formation and studies on understanding their origin in the cosmological context has been the study of previous work (Merritt 1985; Dubinski 1998).

The qualitative picture that emerges in a hierarchical cosmological framework is that during cluster collapse subgroups which contain galaxies merge to form ellipticals. The ellipticals along with unmarred spirals fall into the relaxing cluster potential and settle onto their final orbits. During the initial collapse phase, a large number of galaxies merge in the center to form a the giant elliptical. An additional complication is that additional tidal interactions between 
galaxies and with the galaxy cluster potential itself can lead to serious distortion, stripping and heating of material in many galaxies that fall in (Moore et al. 1996). This leads to two effects - a distorted and heated sub-population of disk galaxies which might be associated with the S0 galaxies and large amount of intracluster stellar debris. The hot intracluster gas can also remove the gas from spirals through ram-pressure stripping and this also has something to do with the formation of the S0 population (e.g. Abadi et al. 1999).

In this talk, I would like to discuss some attempts to study some of the dynamical processes in quantitative detail and the resultant properties of the merger remnants that we identify with regular and giant elliptical galaxies.

\section{Methods}

We have carried out a series of $9 \mathrm{~N}$-body experiments of clusters each containing hundreds of resolved disk galaxies to explore the implications of tidal interaction and merging on the evolution of this population. A simplifying hypothesis is that prior to cluster collapse the galaxies that form are predominantly spirals which have depleted more than half of their gas to form stars by $z=3$ or so. These spirals form in groups which then merge dissipationlessly to form the ellipticals and then fall into a cluster. It is fairly straightforward to set-up pure, N-body experiments to study this process and we use the following procedure.

We first run a cosmological $\mathrm{N}$-body simulation containing dark matter only in a $40 h^{-1} \mathrm{Mpc}$ box. We identify dark matter halos of group and cluster scales with LOS velocity dispersions ranging from $200-600 \mathrm{~km} \mathrm{~s}^{-1}$ at $z=0$ and label the particles that lie within the virial radius. We then re-examine the matter at earlier times around $z=3$ about the epoch of galaxy formation and identify isolated dark halos which we identify with galaxies. We measure the mass profiles of these halos and extract the maximum circular velocity and virial mass. These quantities are used to scale an $\mathrm{N}$-body realization of a spiral galaxy containing a disk, bulge and dark halo. The spiral galaxy model is used is Model B from Kuijken and Dubinski (1995) which is designed to be like an Sb galaxy with an exponential disk and rotation curve similar to the Milky Way. We scale the model by assuming disk mass traces light and that the Tully-Fisher relation holds for the measured circular velocity. The disk scale lengths for the models is set assuming Freeman's law i.e. constant central surface brightness. These assumptions produce typically spirals ranging in luminosity from about 0.05-1 $L_{*}$ with disk scale-lengths from 1-10 kpc. We have carried out 9 simulations where we have replaced 300 dark halos with disk galaxies scaled in this way. Each disk+bulge is resolved with 12000 stars and surrounded by an 8000 particle halo. This locally increases the resolution from 10-100 times above the pure dark matter simulation. The results discussed below assumed a standard cold dark matter universe $(\Omega=1, \Lambda=0.0$ and $h=0.5)$. Although, the cosmological parameters now favoured are quite different than these we believe the basic dynamical results will not be affected except for perhaps the timing of certain events. In any event, we are now carrying out experiments in the currently favoured cosmological models. 


\section{Results}

\subsection{General Observations}

These simulations allow us to follow the dynamical evolution of a population of 2700 disk galaxies as they fall into clusters. We first note that the final number of galaxies is approximately halved because of merging. The number of galaxies we count in and around clusters goes from 2700 at $z=3$ to about 1500 by $z=0$. We developed an automated procedure based on the dynamical evolution to classify the galaxies as spirals, ellipticals, or harassed spirals. We assumed that any observed galaxy that accreted more than $25 \%$ in one merging event is an elliptical. We defined spirals as those galaxies that suffered no major mergers. Finally, harassed spirals were those galaxies that had not suffered a major merger but nevertheless lost more than half of their disk mass presumable to tidal stripping. These classifications are fairly arbitrary but reasonable and lead to the population divided among $55 \%$ spirals, $25 \%$ ellipticals and a surprising $20 \%$ harassed spirals for the simulations. If we make the assumption that the harassed spirals can be identified with the S0 population we see that half the galaxies are early types in and nearby clusters in approximate accord with the observed morphology-density effect. The number of ellipticals remains fairly constant with time from $z=2$ to $z=0.0$ though the number of regular spirals decreases through merging and transformation to harassed spirals. One might naively think that the number of ellipticals should be growing in time but this does not seem to be the case. It appears that the roughly constant number suggests that a base population of ellipticals forms early on by $z=2$ and while mergers continue the numbers do not grow since some ellipticals in fact merge with other galaxies to form larger ellipticals.

\subsection{A Numerical Fundamental Plane}

These simulations produce more than 300 ellipticals at modest numerical resolution with approximately 30k-100k "stars" per galaxy. This sample is large enough to examine fundamental plane properties of merger remnants for direct comparison to real populations. We culled this population down to about 160 galaxies discarding mainly those objects which were still in the state of merging or not fully relaxed as revealed by irregular surface brightness profiles and those objects with small effective radii that may have been affected by our limited numerical resolution. We tried to be faithful to current observational techniques applied to ellipticals to determine the the three parameters of effective radius $R_{e}$, mean surface brightness within $R_{e}$ and central velocity dispersion. For real galaxies, these quantities are found to be closely confined to a plane in this 3-space following the relation:

$$
\log R_{e}=\alpha \log \sigma+\beta \log I_{e}+\text { const. }
$$

where $\alpha=1.53 \pm 0.08$ and $\beta=-0.79 \pm 0.03$ for elliptical galaxies observed in the near infrared (Pahre et al. 1998) and values around $\alpha=1.24 \pm 0.07$ and $\beta=-0.82 \pm .02$ in the optical (Jørgensen et al. 1996). If galaxies were perfectly homologous and in virial equilibrium we would expect $\alpha=2$ and $\beta=-1.0$. Ellipticals are probably in virial equilibrium so the deviation from 


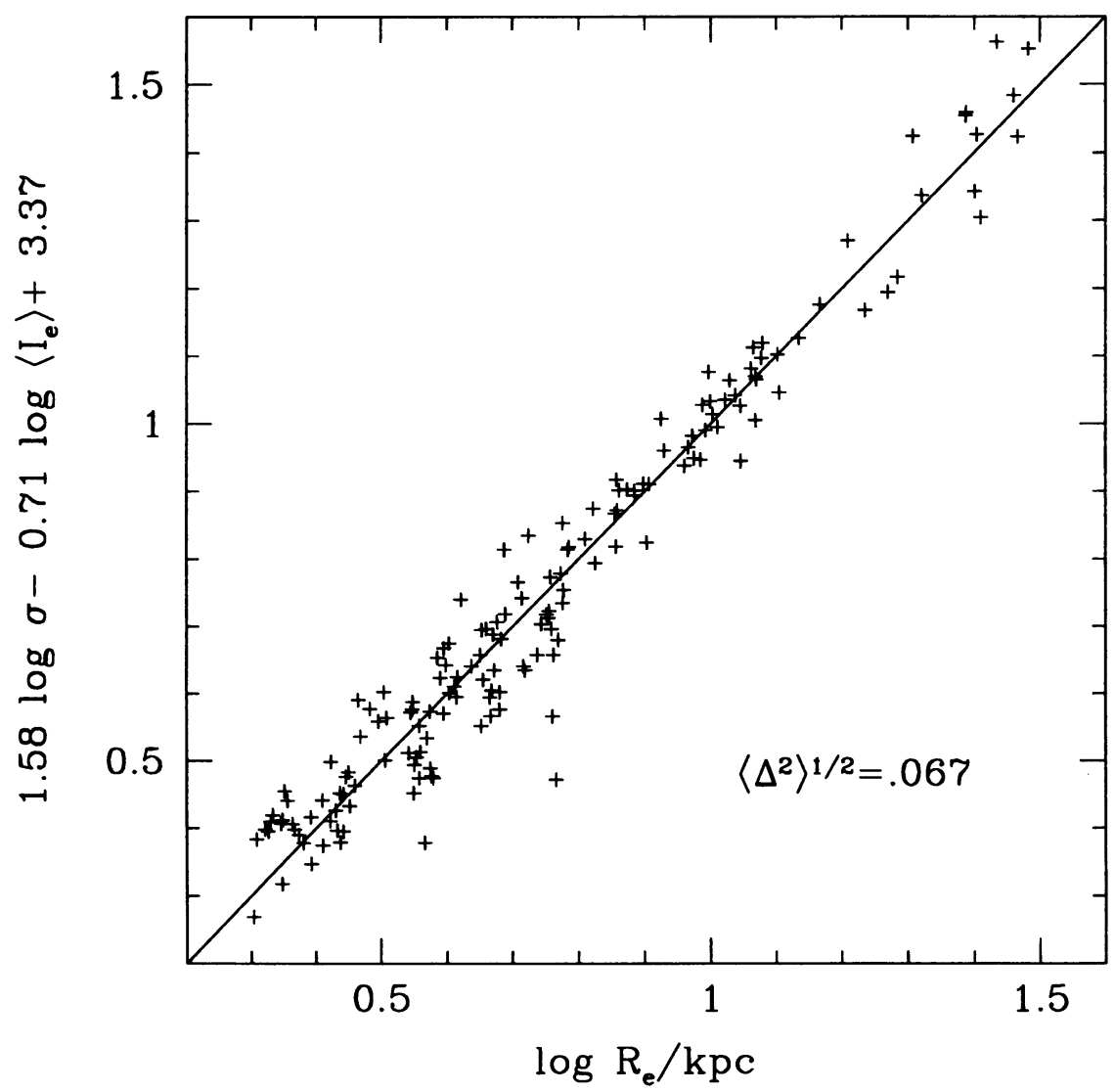

Figure 1. Fundamental plane as measured from a sample of 162 merger remnants.

the fundamental plane suggests some non-homology among the population or some change in properties with scale.

The fundamental plane for the numerical sample has $\alpha=1.58$ and $\beta=$ -0.71 (Figure 1). The most appropriate sample to compare our results to are the near-infrared observations since measurements of the surface brightness in this band probably corresponds most closely to the surface density of stars that we measure for this comparision. While the agreement is not perfect the coefficients are remarkably close to real values and the relation is as tight with an rms deviation of only 0.07 dex.

We had no prior expectations that our simulated sample would settle to a fundamental plane but apparently it has. We can draw the following conclusion: a population of regular spiral galaxies that have scaling properties determined by the Tully-Fisher relation that merge within the hierarchical framework of 
a dark matter cosmological scenario will naturally settle into a fundamental plane. Work on idealized merging hiearchies also suggests an attractor to a fundamental plane (Capelato et al. 1995). Perhaps since the spirals themselves are non-homologous they pass this characteristic on to their merged descendants. The problem then might be reduced to finding the source of the Tully-Fisher relation alone. We are still trying to understand the source of the non-homology in the merger remnants and attempting to redo similar experiments at higher resolution to convince ourselves of the affect.

\section{Brightest Cluster Galaxies}

All nine cluster simulations end up with a giant elliptical galaxy at the bottom of the cluster potential well (Figure 2) confirming the picture laid out in Dubinski (1998). These giant ellipticals can be identified with the brightest cluster galaxies (BCG's) seen in most clusters. They are the product of the merger of typically 10 or more galaxies some spiral and some ellipticals that formed in previous mergers. Most of their mass is built up early in the history of the cluster.

We measured the surface brightness and velocity dispersion profiles for quantitative comparisons to real BCG's (Figure 3.). The simulations show that the light profiles extend all the way out to the virial radius so generically clusters should be filled with intracluster stars which are the remnants of merged and stripped spirals. Intracluster stars have been detected in the Virgo cluster both through direct observation of red giants (Ferguson et al. 1998) and planetary nebulae (e.g. Feldmeier et al. 1998). These stars are seen to arise naturally during the formation of the giant elliptical and the tidal stripping of spirals. We are in the process of quantifying the amount of intracluster light precisely but the rough answer is that approximately $20 \%$ of cluster stellar mass is in the diffuse light of these stars below the Holmberg limit of $\mu_{B}=26.5$. There are no bona fide $\mathrm{cD}$ galaxies formed with envelopes that deviate significantly from the $R^{1 / 4}$ law at large radius. The spikes in the profiles come from the light of individual galaxies within the cluster but you can see that without these spikes the trend is towards an $R^{1 / 4}$ out to very large radii and low surface brightness. The veracity of $\mathrm{cD}$ envelopes has been questioned recently in some careful measurements of diffuse light in Abel 1651 by Gonzalez et al. 2000. They find that the light profile is consistent with a deVaucouleurs profile out to about one third of the virial radius with no sign of a $\mathrm{cD}$ envelope. This observation is consistent with what we see in these simulations.

Finally, we also measured the central velocity dispersion of the simulated BCG's. The lower mass clusters had dispersions of $200-300 \mathrm{~km} / \mathrm{s}$ but the largest clusters had velocity disperions as high as $500 \mathrm{~km} / \mathrm{s}$ - somewhat greater than what is typically observed. The origin of the discrepancy might lie with our choice of initial conditions. We assumed well-formed field spirals following current epoch scaling relations. It seems likely that early populations of spirals might be more compact since they will not have accreted all of their gas. Once falling into clusters accretion infall would stop because of tidal truncation of the galaxies by the forming cluster potential. The material stripped from more tightly bound galaxies could possibly have a lower velocity dispersion since it would result in a more centrally concentrated population that the cluster dark 


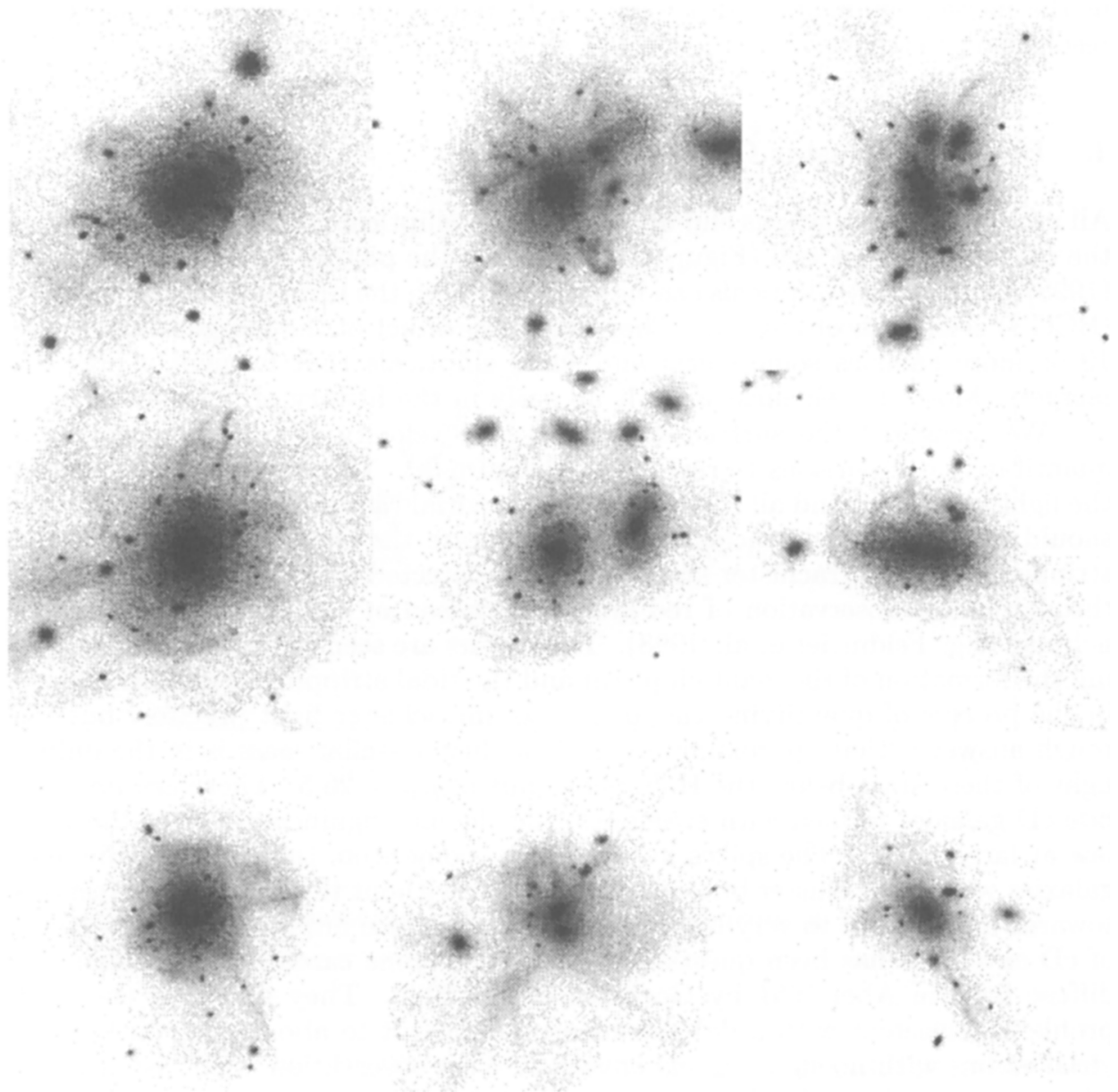

Figure 2. Nine simulated clusters showing the brightest cluster galaxy with orbiting galaxies and surrounding tidal debris. The boxes go out to the virial radius for each cluster which corresponds to about $1 \mathrm{Mpc}$ for these systems. 
halo. We tested this hypothesis by isolating the bulges from each of the progenitor galaxies and measuring the velocity dispersion of the bulge stars alone in the final giant elliptical. As expected, the velocity dispersion was lower for the bulge stars compared to the disk stars. This reduced the central velocity dispersion in the worst case by about $20 \%$ to around $400 \mathrm{~km} / \mathrm{s}$. This velocity is still high but perhaps a combination of this effect plus some dissipation in the core could explain the lower velocity dispersion seen in real BCG's.

\section{Conclusions}

The simple hypothesis that ellipticals come from the mergers of ordinary spiral galaxies in a cosmological framework leads to a picture with many strengths. We can explain the morphology-density relationship in clusters, we get a fundamental plane scaling relationship for free, uncover a compelling scenario for the formation of the BCG's as well as describe their surface brightness profiles and amount of intracluster light. The only deficiency is a excessive central velocity dispersion but this may be the result of over-simplified initial conditions. Future calculations should attempt to increase the dynamical resolution to check for convergence and of course more rigorous models including gas dynamics and star formation should be explored in this context to understand the effects of dissipation and more realistic timeframes for galaxy formation.

Acknowledgments. The authors acknowledge the National Center for Supercomputing Applications where these calculations were carried out. JJD acknowledges NSERC.

\section{References}

\section{References}

Abadi, M.G., Moore, B. \& Bower, R.G. 1999, MNRAS, 308, 947

Capelato, H.H, de Carvalho, R.R., \& Carlberg, R.G. 1995, ApJ, 451, 525

Dressler, A. 1980, ApJ, 236, 351

Dressler, A. 1984, ARA\&A, 22, 185

Dubinski, J. 1998, ApJ, 502, 141

Feldmeier, J.J., Ciardullo, R. \& Jacoby, G. 1998, ApJ, 503, 109

Ferguson, H.C., Tanvir, N.D. \& von Hippel, T. 1998, Nature, 391, 461

Gonzalez, A., Zabludoff, A., Zaritsky, D. \& Dalcanton, J. 2000, ApJ, 536, 561

Jørgenson, I., Franx, M., \& Kjærgaard, P. 1996, MNRAS, 280, 167

Kuijken, K. \& Dubinski J. 1995, MNRAS, 277, 1341

Merritt, D. 1985, ApJ, 289, 18 Moore, B., Katz, N., Lake, G., Dressler, A., \& Oemler, A.,Jr 1996, Nature, 379, 613

Pahre, M.A., Djorgovski, S.G., and De Carvalho, R.R., AJ, 116, 1591 

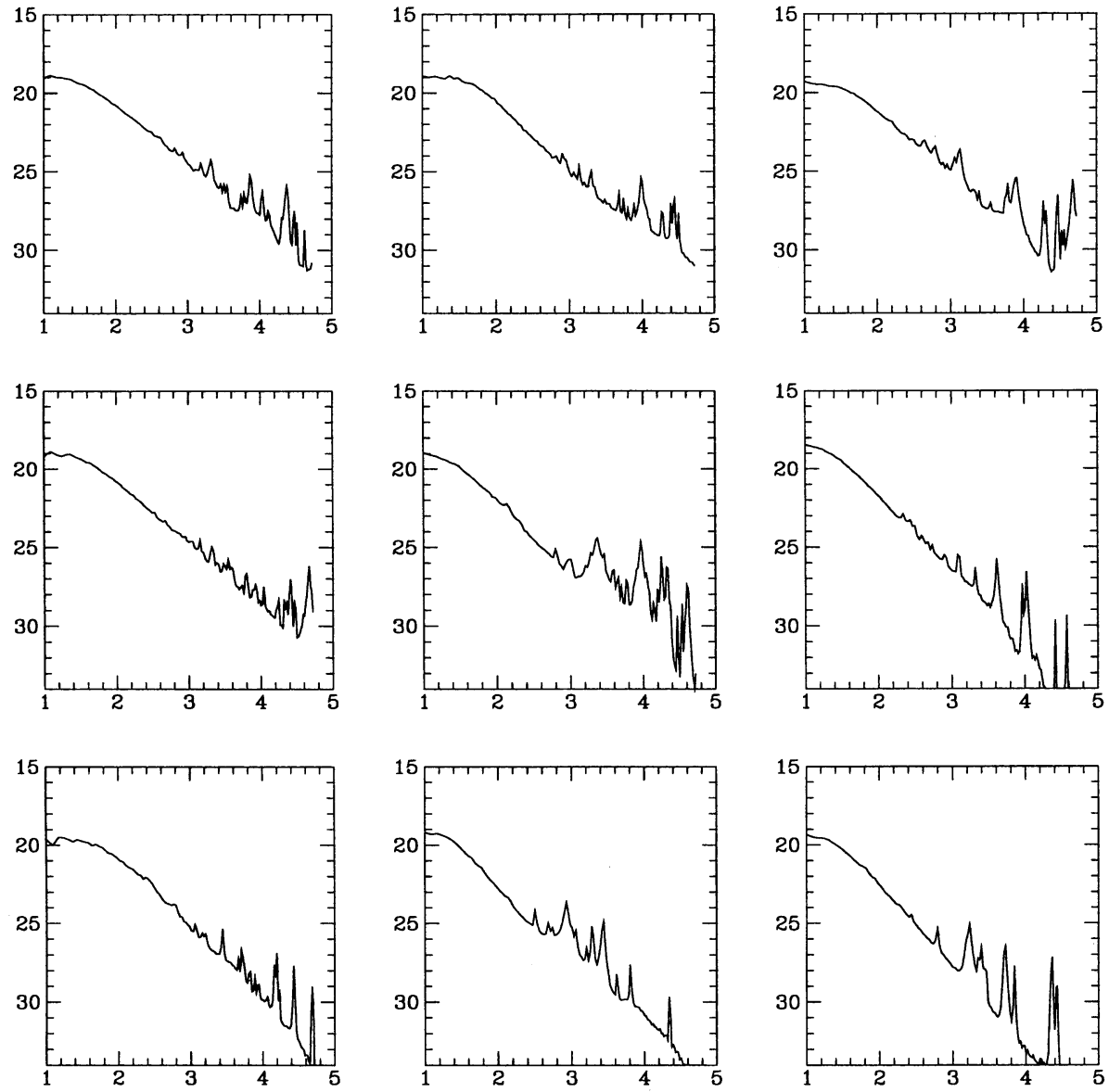

Figure 3. Light profiles of the 9 simulated clusters. The axes are surface brightness $\mu$ in mag. per square arcsec and $(R / 1 k p c)^{1 / 4}$. We assume that the stars have an intrinsic mass-to-light ratio of 3 . 